\title{
MAPK/AP-1 pathway regulates benzidine-induced cell proliferation through the control of cell cycle in human normal bladder epithelial cells
}

\author{
LI ZHAO $^{1 *}$, TAO ZHANG ${ }^{2 *}$, HAO GENG $^{2}$, ZHI-QI LIU ${ }^{2}$, ZHAO-FENG LIANG $^{3,4}$, \\ ZHI-QIANG ZHANG ${ }^{2}$, JIE MIN ${ }^{2}$, DE-XIN YU ${ }^{2}$ and CAI-YUN ZHONG ${ }^{3}$

\begin{abstract}
${ }^{1}$ Department of Urology, Affiliated Fuyang Hospital of Anhui Medical University, Fuyang, Anhui 236000;
${ }^{2}$ Department of Urology, The Second Affiliated Hospital of Anhui Medical University, Hefei, Anhui 230032;

${ }^{3}$ Department of Nutrition and Food Safety, School of Public Health, Nanjing Medical University, Nanjing, Jiangsu 211166;

${ }^{4}$ Department of Medical Examination, School of Medicine, Jiangsu University, Zhenjiang, Jiangsu 212000, P.R. China
\end{abstract}

Received April 21, 2016; Accepted June 27, 2017

DOI: $10.3892 / \mathrm{ol} .2018 .9155$

\begin{abstract}
Bladder cancer is the most common malignancy of the urinary tract. Long-term exposure to benzidine is one of the major causes of bladder cancer. However, the mechanism of benzidine-induced bladder cancer is not yet sufficiently characterized. Dysregulated cell proliferation serves a critical role in cancer initiation and development; whether benzidine promotes cell proliferation, and the role of MAPKs in this process, have not previously been investigated. The present study aimed to investigate the benzidine-induced modulation of intracellular mitogen-activated protein kinases (MAPKs) and activator protein-1 (AP-1) signaling cascades on cell proliferation in SV-40 immortalized human uroepithelial cells (SV-HUC-1). It was identified that benzidine exposure enhanced the proliferation of SV-HUC-1 cells, promoted the transition of cells from $\mathrm{G} 1$ to $\mathrm{S}$ phase and altered the expression level of cell cycle-associated genes at the mRNA and protein levels. Furthermore, exposure of the SV-HUC-1 cells to benzidine was associated with the activation of MAPKs,
\end{abstract}

Correspondence to: Professor De-Xin Yu, Department of Urology, The Second Affiliated Hospital of Anhui Medical University, 80 Feicui Road, Hefei, Anhui 230032, P.R. China

E-mail: yudx_urology@126.com

Professor Cai-Yun Zhong, Department of Nutrition and Food Safety, School of Public Health, Nanjing Medical University, 818 East Tianyuan Road, Nanjing, Jiangsu 211166, P.R. China

E-mail: cyzhong@njmu.edu.cn

*Contributed equally

Abbreviations: SV-HUC-1, SV-40 immortalized human uroepithelial cell

Key words: bladder cancer, benzidine, cell proliferation, mitogen activated protein kinase/activator protein-1, SV-HUC-1 cells including extracellular regulated protein kinases 1 and 2, p38 and Jun N-terminal kinase. The downstream target of MAPKs, AP-1 monomers, was also activated. Benzidine-induced proliferation was reversed by MAPK-specific inhibitors. Thus, the present study demonstrated that benzidine enhances the proliferation of bladder cells via activating the MAPK/AP-1 pathway, which may provide novel insights into the molecular mechanisms of benzidine-initiated bladder tumorigenesis, as well as cancer prevention.

\section{Introduction}

Bladder cancer is the most common malignancy of the urinary tract (1). There were an estimated 16,000 mortalities due to bladder cancer in 2015 in the United States alone (2). A notable risk factor for the development of bladder cancer is the occupational exposure to aromatic amines (e.g., benzidine, 2-naphthylamine and 4-aminobiphenyl). Benzidine is commonly encountered in the industrial dye and rubber industries, as well as in hair dyes, paints, fungicides, motor vehicle exhaust fumes and industrial pollutant emissions (3). Thus, benzidine and other aromatic compounds are notable hazards for human health. Although there have been a number of epidemiological investigations about the association of benzidine exposure with bladder cancer and other carcinomas (4), including our previous study, in which it was demonstrated that benzidine could induce the epithelial-mesenchymal transition in human uroepithelial cells through the extracellular regulated protein kinases 1 and 2 (ERK1/2) pathway (5), limited research has been conducted to investigate whether benzidine exposure promotes cell proliferation and the underlying mechanisms of this process.

The cell cycle is the process through which living cells replicate genetic information and generate progeny cells. The process can be divided into two highly regulated phases: The interphase and mitotic phase. The former involves three phases, the G1, S and G2 phases. Complex mechanisms are associated with the modulation of the cell cycle, which is strictly regulated in normal cells; modifications to cell cycle 
regulation may lead to disease, including tumor formation (6). Cell cycle dysregulation is necessary for cancer initiation and progression (7).

The mitogen-activated protein kinases (MAPKs), a family of enzymes that catalyze the phosphorylation of specific serine and threonine residues on target substrates in order to convert extracellular signals to intracellular, include four major subfamilies: ERK1/2, the Jun N-terminal kinases (JNKs), p38 and ERK5 $(8,9)$. MAPKs serve important functions in many life processes, including proliferation, differentiation and apoptosis (10). The activation of the ERK1/2, p38, and JNK/MAPK pathways is associated with the induction of many transcription factors, resulting in the altered expression of various genes associated with tumor cell proliferation, apoptosis, angiogenesis, metastasis and the progression of various types of cancer, including bladder cancer $(11,12)$.

In the present study, the mechanism of benzidine-induced SV-HUC-1 cell proliferation was investigated, including the role of MAPKs in the proliferation process. The aim was to elucidate a potential mechanism for benzidine-induced carcinogenesis.

\section{Materials and methods}

Chemicals and reagents. Benzidine (4,4'-diaminobiphenyl; $\geq 98.0 \%$ ), dimethyl sulfoxide (DMSO), MTT, methanol, glutaraldehyde and all other chemicals and dyes of the highest purity were purchased from Merck KGaA (Darmstadt, Germany). The kinase inhibitors U0126 (against ERK1/2), SB203580 (against p38) and SP600125 (against JNK) were obtained from Cell Signaling Technology, Inc. (Danvers, MA, USA). Ham's F12 medium, fetal bovine serum (FBS), PBS, antibiotics and trypsin were obtained from HyClone (GE Healthcare, Chicago, IL, USA). Antibodies to ERK1/2 (cat. no. BS-2637R; 1:1,000), p38 (cat. no. 12; 1:1,000), JNK (cat. no. AR2045; 1:1,000), phosphorylated (p)-ERK1/2 (cat. no. RS-2637S; 1:1,000), p-JNK (cat. no. AF3318; 1:1,000), p-p38 (cat. no. 9212; 1:1,000), p-c-Jun (cat. no. AF-3095; 1:1,000), p-c-Fos (cat. no. 5348; 1:1,000), cyclin D1 (cat. no. 2978; 1:1,000), proliferating cell nuclear antigen (PCNA; cat. no. 10205-2-AP; 1:1,000) and P21 (cat. no. 10355-1-AP; 1:1,000) were purchased from Cell Signaling Technology, Inc. The GAPDH (cat. no. 60004-1-Ig; 1:1,000) antibody was obtained from Biogot Technology Co., Ltd. (Nanjing, China). Monoclonal rabbit Jun D antibody (cat. no. sc-74; 1:1,000), Jun B antibody (cat. no. 10486-1-AP; 1:1,000), Fra-1 antibody (cat. no. D80B4; 1:1,000) and FosB antibody (cat. no. AF5010; 1:1,000) was purchased from Santa Cruz Biotechnology, Inc. (Dallas, TX, USA). Mouse anti-rabbit IgG secondary antibodies (cat. no. bs-0295M; 1:10,0000) were purchased from Beyotime Institute of Biotechnology (Nanjing, China).

Cell culture and treatments. SV-40 immortalized human uroepithelial cells (SV-HUC-1) were obtained from the American Type Culture Collection (Manassas, VA, USA) and grown in $25-\mathrm{cm}^{2}$ flasks (initial density, $1 \times 10^{5}$ cells $/ \mathrm{ml}$ ). Cells were maintained in Ham's F-12 medium supplemented with $10 \%$ FBS, 100 units $/ \mathrm{ml}$ penicillin and 100 units $/ \mathrm{ml}$ streptomycin at $37^{\circ} \mathrm{C}$ with $5 \% \mathrm{CO}_{2}$ in a humidified incubator. Following culture for $12 \mathrm{~h}$, cells were exposed to different concentrations of benzidine (including 0, 0.0001, 0.001, 0.005, $0.01,0.05,0.1,1,10,50,75,100,125,150$ and $200 \mu \mathrm{M})$ diluted with DMSO, and/or treated with U0126 (10 $\mu \mathrm{M})$, SB203580 $(10 \mu \mathrm{M})$ or SP600125 $(5 \mu \mathrm{M})$, and were passaged daily for 6 days. All experiments were performed three times.

Cell proliferation assay. Cell viability was assayed by MTT conversion to formazan. Subsequent to growing to $80 \%$ confluence on a $10 \mathrm{~cm}^{2}$ plate, $5 \times 10^{4} \mathrm{SV}$-HUC-1 cells per well were seeded in 96-well plates. Then cells were treated with $100 \mu \mathrm{l}$ growth medium with $0.1 \%$ DMSO or benzidine $(0.001-200 \mu \mathrm{M})$ for 2 or 6 days. MTT solution (10 $\mu \mathrm{l}$ of $5 \mathrm{mg} / \mathrm{ml})$ was added to each well and the plates incubated for an additional $4 \mathrm{~h}$ at $37^{\circ} \mathrm{C}$. The medium was removed and DMSO was added to each well to solubilize precipitants. Absorbance was measured at $490 \mathrm{~nm}$ using a microplate reader. All measurements were performed in triplicate.

Western blotting. For the western blot analysis, $6 \times 10^{6}$ SV-HUC-1 cells per dish were seeded in $100 \mathrm{~mm}$ plastic tissue culture dishes. Following culture for $12 \mathrm{~h}$ as previously described, cells were either exposed to different concentrations of benzidine $(0,0.001,0.005,0.01,0.05$ or $0.1 \mu \mathrm{M})$ or treated with U0126, SB203580 or SP600125 for 6 days. Cells were harvested, washed with ice-cold PBS, and lysed in RIPA buffer (Thermo Fisher Scientific, Inc., Waltham, MA, USA). Concentrations of the precipitated proteins in cell lysates were measured with BCA Protein Assay (Pierce; Thermo Fisher Scientific, Inc.). Then, proteins (50 g per lane) were separated by $10 \%$ SDS-PAGE and transferred onto a polyvinylidene difluoride membrane (EMD Millipore, Billerica, MA, USA). Subsequent to blocking in 5\% fat-free dry milk in Tris-buffered saline with Tween-20 (TBST), membranes were incubated with primary antibodies (1:500, diluted with $5 \%$ milk) overnight at $4^{\circ} \mathrm{C}$, washed in TBST and then incubated with goat anti-rabbit peroxidase-conjugated secondary antibodies (1:500, diluted with 5\% milk) for $1 \mathrm{~h}$ at room temperature. The blots were subsequently developed using an enhanced chemiluminescence detection kit (Amersham; GE Healthcare) and exposed to film (Kodak, Rochester, NY, USA). GAPDH served as the loading control. For densitometric analyses, protein bands on the blots were measured using the Eagle Eye II software (Agilent Technologies, Inc., Santa Clara, CA, USA).

Reverse transcription-quantitative polymerase chain reaction (RT-qPCR). For mRNA analysis, 6x106 SV-HUC-1 cells per dish were seeded in $100-\mathrm{mm}$ plastic tissue culture dishes. Following culture for $12 \mathrm{~h}$, cells were exposed to $0,0.001$, $0.005,0.01,0.05$ or $0.1 \mu \mathrm{M}$ benzidine. Following a further 6 days of culture, cells were harvested and total RNA was isolated with RNAiso Plus (Takara Bio, Inc., Otsu, Japan) following the manufacturer's protocol. Total RNA was transcribed into cDNA using AMV reverse transcriptase (Takara Bio, Inc.) according to the manufacturer's protocol.

qPCR was performed using the Power SYBR-Green Master Mix (Takara Bio, Inc.) and an Applied Biosystems 7300 Real-Time PCR Detection System (Thermo Fisher Scientific, Inc.). The $20 \mu 1$ total reaction mixture included $10 \mu \mathrm{l} \mathrm{SYBR}$

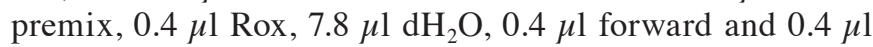



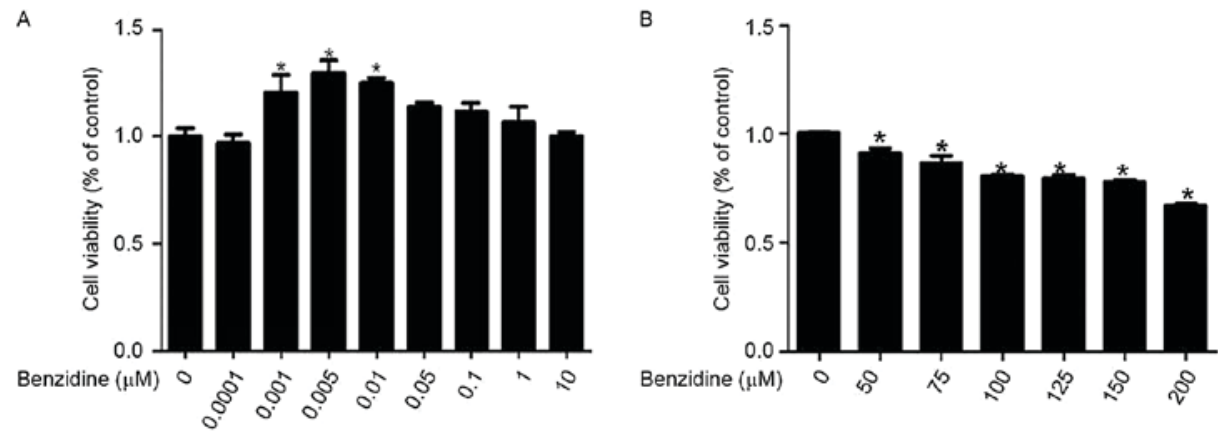

Figure 1. Detection of SV-HUC-1 cell viability following treatment with various concentration of benzidine. (A) SV-HUC-1 cells were treated with benzidine for 6 days. The lowest concentration of benzidine $(0.0001 \mu \mathrm{M})$ had no effect on cell viability, whereas benzidine at concentrations from 0.001 to $0.01 \mu \mathrm{M}$ enhanced SV-HUC-1 cell viability. Doses higher than $0.01 \mu \mathrm{M}$ also elevated cell viability, although no statistical significance was determined. (B) SV-HUC-1 cells were treated with high doses of benzidine for $48 \mathrm{~h}$. The higher concentrations of benzidine $(>10 \mu \mathrm{M})$ exhibited a toxic effect on SV-HUC-1 cells Values represent the mean \pm standard deviation of triplicate measurements. ${ }^{*} \mathrm{P}<0.05$ compared with the $0 \mu \mathrm{M}$ group. SV-HUC-1, SV-40 immortalized human uroepithelial cells.

reverse primers, and $1 \mu \mathrm{l}$ cDNA sample. Each sample was repeated three times.

All primers were synthesized by Invitrogen (Thermo Fisher Scientific, Inc.). The primers used were: Cyclin D1 forward, 5'-CGTGGCCTCTAAGATGAAGG-3' and reverse, 5'-TGCGGATGATCTGTTTGTTC-3'; p21 forward, 5'-GAC ACCACTGGAGGGTGACT-3' and reverse, 5'-CAGGTCCAC ATGGTCTTCCT-3'; PCNA forward, 5'-CTGAAGCCGAAA CCAGCTAGACT-3' and reverse, 5'-TCGTTGATGAGGTCC TTGAGTGC-3'; GAPDH forward, 5'-GCTGCCCAACGC ACCGAATA-3' and reverse, 5'-GAGTCAACGGATTTGGTC GT-3'. The PCR program included an initial denaturation step at $95^{\circ} \mathrm{C}$ for $15 \mathrm{sec}$, followed by 40 cycles of amplification and quantification at $95^{\circ} \mathrm{C}$ for $10 \mathrm{sec}, 60^{\circ} \mathrm{C}$ for $30 \mathrm{sec}$, and $72^{\circ} \mathrm{C}$ for $30 \mathrm{sec}$. At the end of the program, a melting curve analysis was performed. Fold changes in the expression of each gene were calculated using the comparative quantitation cycle $(\mathrm{Cq})$ method using the formula $2^{-\Delta \Delta C q}(13)$.

Cell cycle analysis. SV-HUC-1 (1×10 cells/well) were grown in 6-well plates followed by treatment with $0,0.001,0.005$, $0.01,0.05$ or $0.1 \mu \mathrm{M}$ benzidine. Following 6 days of growth, cells were trypsinized, washed twice with cold PBS, and centrifuged $\left(500 \mathrm{x} \mathrm{g}\right.$ for $5 \mathrm{~min}$ at $\left.4^{\circ} \mathrm{C}\right)$. The cell pellet was resuspended in $500 \mu \mathrm{l}$ cold PBS and fixed in 2-3 $\mathrm{ml} 70 \%$ ethanol at $4^{\circ} \mathrm{C}$ for $1-14$ days. Cells were centrifuged $(500 \mathrm{x} \mathrm{g}$ for $5 \mathrm{~min}$ at $4^{\circ} \mathrm{C}$ ) and resuspended in $500 \mu \mathrm{lPBS}$. Propidium iodide staining buffer was added in the dark at room temperature for $30 \mathrm{~min}$ and cells were then analyzed with flow cytometry (FACStar cytofluorometer; BD Biosciences, Franklin Lakes, NJ, USA). Each assay was repeated three times.

Statistical analysis. Statistical analyses were performed with SPSS 17.0 (SPSS, Inc., Chilcago, IL, USA). All data are expressed as mean \pm standard deviation. One-way analysis of variance or the Kruskal-Wallis test were used to analyze differences among groups. In case of comparison between two groups, an unpaired Student's t-test was used. Results are expressed as mean \pm standard deviation from $\geq 3$ independent experiments. $\mathrm{P}<0.05$ was considered to indicate statistically significant differences.

\section{Results}

Benzidine enhances the proliferation of SV-HUC-1 cells. The effect of benzidine on the proliferation of SV-HUC-1 cells was determined by an MTT assay. The data revealed that treatment with benzidine at the concentration range of $0.001-0.01 \mu \mathrm{M}$ for 6 days significantly increased the cell viability of SV-HUC-1 cells compared with the $0 \mu \mathrm{M}$ control group ( $\mathrm{P}<0.05$; Fig. 1A), whereas treatment with benzidine at doses $>10 \mu \mathrm{M}$ induced a toxic effect on SV-HUC-1 cells (Fig. 1B). Therefore, benzidine at doses from $0.001-0.1 \mu \mathrm{M}$ was used in subsequent experiments.

Benzidine facilitates SV-HUC-1 cell transition from G1 to $S$ phase. To ascertain that benzidine treatment induced SV-HUC-1 cell proliferation as demonstrated by the MTT assay data, flow cytometry was applied to detect alterations in the cell cycle distribution. Following 6 days of treatment with benzidine, an increased percentage of cells in the $\mathrm{S}$ and G2 phases was observed (Fig. 2A and B). The fraction of cells in the $\mathrm{S}$ phase increased from 40.88 to $48.81 \%(\mathrm{P}=0.036)$ and the fraction of cells in the $\mathrm{G} 2$ or $\mathrm{M}$ phase increased from 13.53 to $19.50 \%(\mathrm{P}=0.018)$; the population of cells in the $\mathrm{G} 1$ phase decreased from 45.59 to $34.22 \%(\mathrm{P}<0.01)$.

Benzidine alters cell cycle associated marker expression in SV-HUC-1 cells. The protein and mRNA levels of cyclin D1, p21 and PCNA were measured as cell cycle-specific markers. The results demonstrated that exposure to benzidine significantly increased the protein levels of cyclin D1 and PCNA (both $\mathrm{P}<0.01,0.001-0.1 \mu \mathrm{M}$ ), whereas the $\mathrm{p} 21$ protein level was decreased $(\mathrm{P}<0.01,0.05-0.1 \mu \mathrm{M}$; Fig. 3A and $\mathrm{B})$. The mRNA levels of cyclin D1 $(\mathrm{P}<0.01,0.001-0.05 \mu \mathrm{M})$ and PCNA $(\mathrm{P}<0.01,0.005-0.1 \mu \mathrm{M})$ were also significantly elevated, whereas the p21 mRNA was downregulated $(\mathrm{P}<0.01$, 0.001-0.1 $\mu \mathrm{M}$; Fig. 3C).

Exposure to benzidine induces MAPK/AP-1 activation. To determine whether MAPK/AP-1 signaling pathways were activated in benzidine-mediated SV-HUC-1 proliferation, MAPK and AP-1 markers were measured with western blotting. Increases in p-ERK, p-p38 and p-JNK protein levels were 

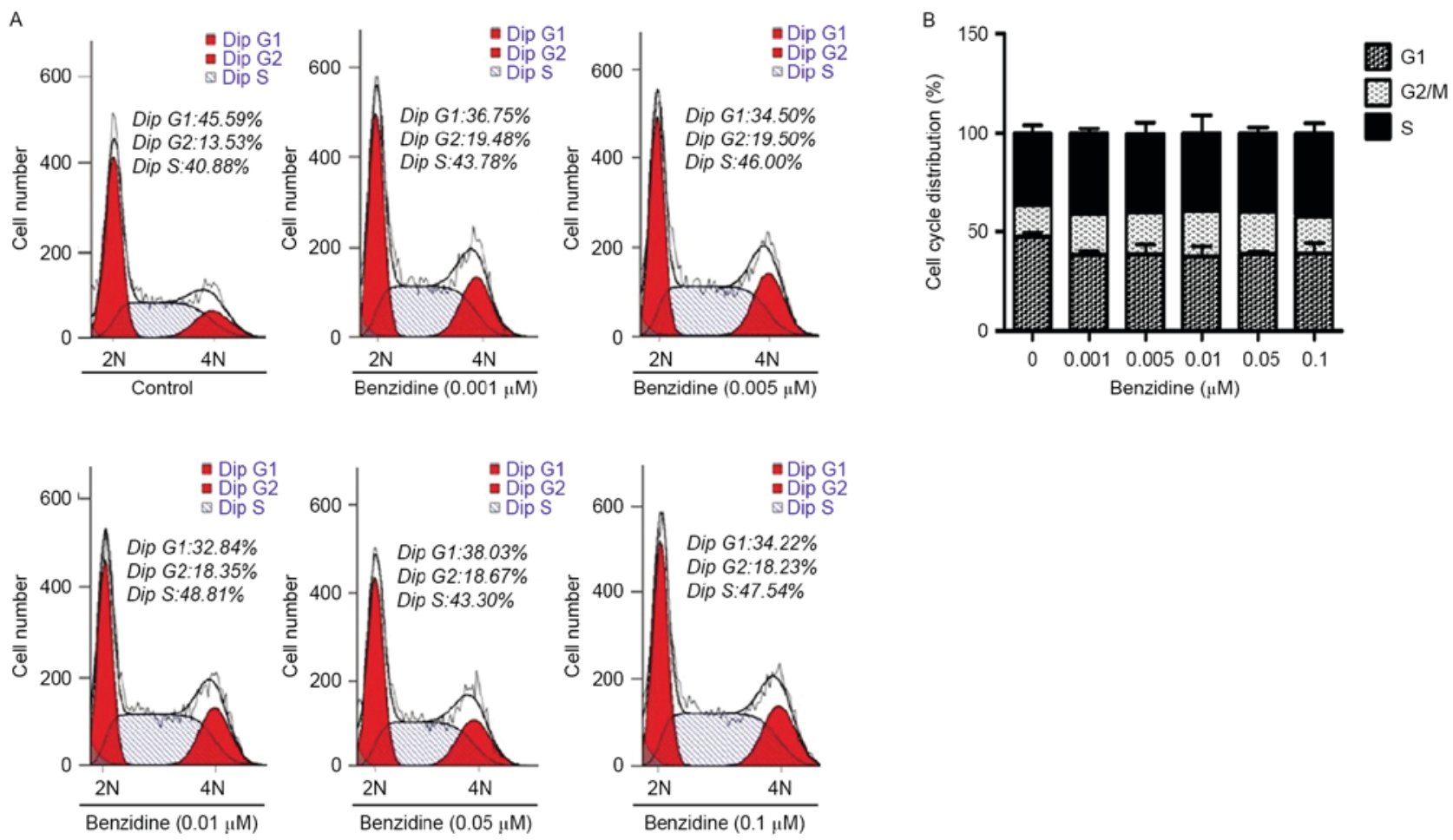

Figure 2. Measurement of benzidine-mediated changes in cell cycle distribution. (A) Flow cytometry was used to analyze SV-HUC-1 cells treated with benzidine $(0.001-0.1 \mu \mathrm{M})$ for 6 days. The proportion of cells in $\mathrm{G} 1$ phase was decreased in the treated groups; the decrease in the $0.01 \mu \mathrm{M}$ group was the largest. Meanwhile, the proportion of cells in S and G2 phase was elevated. (B) Histograms of the data from part A. The data are expressed as the mean \pm standard deviation of three independent experiments. SV-HUC-1, SV-40 immortalized human uroepithelial cells.

observed, whereas total ERK1/2, p38 and JNK protein levels remained unchanged in cells treated with benzidine $(\mathrm{P}<0.05$ vs control group) (Fig. 4A and B). The protein levels of members of the Jun family, including p-c-Jun, JunB and JunD (Fig. 4C and D), and members of the Fos family, including p-c-Fos, Fos-like antigen 1 and FosB (Fig. 4E and F), were also observed to be increased $(\mathrm{P}<0.05)$.

Benzidine-mediated SV-HUC-1 cell proliferation is suppressed by MAPK-specific inhibitors. MAPK specific inhibitors (including U0126 for ERK1/2, SB203580 for p38 and SP600125 for JNK) were used to confirm the association between the activation of MAPKs and benzidine-induced cell proliferation. When the cells were treated with the inhibitors, benzidine-elevated cell viability was reversed (Fig. 5A). The protein level of MAPKs and cell cycle-associated proteins following exposure to benzidine and each of the inhibitors was also assessed. The repression of MAPKs, AP-1 monomers and cell cycle protein expression was detected (Fig. 5B-D). The results indicated the importance of MAPK/AP-1 signaling in benzidine-mediated SV-HUC-1 proliferation.

\section{Discussion}

Bladder cancer is a major cause of cancer-associated mortality worldwide (2). The occupational exposure to benzidine has been established as one of the risk factors for bladder cancer (3). The mechanism for the occurrence and development of benzidine-induced bladder cancer is not yet fully characterized. In the present study, low concentrations of benzidine enhanced SV-HUC-1 cell proliferation. Activation of MAPK/AP-1 signaling was also detected. Furthermore, the regulative role of MAPK signaling in benzidine-induced cell proliferation was confirmed.

The regulation of the cell cycle is necessary for cell growth. The escape from the regulation of the cell cycle may cause unlimited proliferation, which serves a critical role in the initiation and progression of tumorigenesis $(14,15)$. In the present study, it was demonstrated for the first time that low concentrations of benzidine exposure enhanced SV-HUC-1 cell proliferation, elevated cyclin D1 and PCNA expression, decreased the expression of $\mathrm{p} 21$, and provoked the transition of cells from $\mathrm{G} 1$ to $\mathrm{S}$ and $\mathrm{G} 2$ phase. Cyclin D1 is a nuclear protein that serves a pivotal role in cell proliferation and the transition from the G1 to $\mathrm{S}$ phase. Its expression has been implicated in cancer from a number of tissue types and in cultured cells (16). Sun et al (17) demonstrated that CyclinD1 protein may serve a different role in modulating chemoresponses in MCF7 and MDA-MB231 cells. Additionally, Guo et al (18) indicated that Cyclin D1 is a cell cycle machine, a sensor of extracellular signals and serves an important role in G1-S phase progression; their research demonstrated that cyclinD1 is an activator of cell cycle initiation and progression.

PCNA is a non-histone nuclear protein that is necessary for DNA synthesis, and its expression is well documented as enhancing cancer cell proliferation (19). The PCNA gene contains AP-1 sites in the promoter region and its expression is regulated by AP-1 activity. The association of PCNA with cancer transformation resulted in the use of PCNA as a diagnostic and prognostic cell cycle marker for tumors (20). 
A

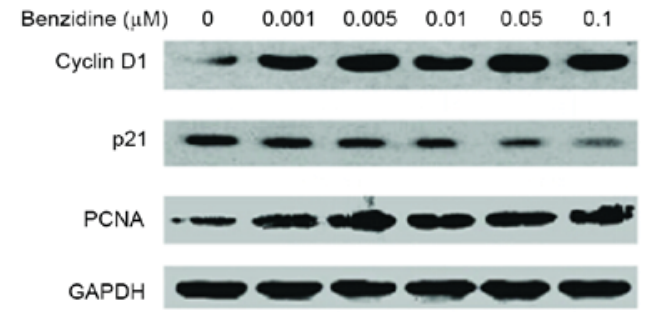

B

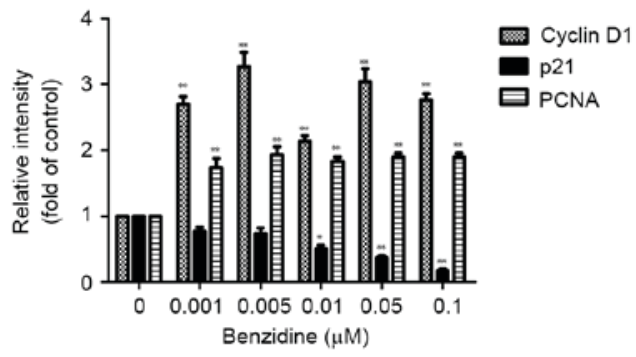

C

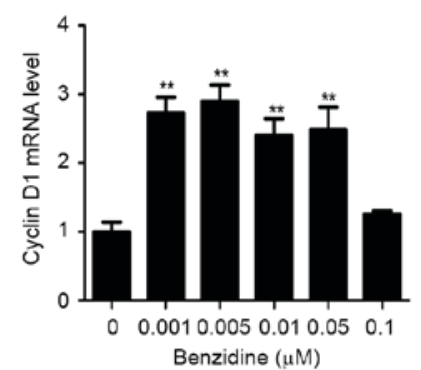

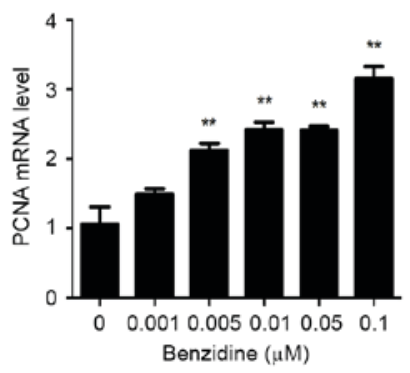

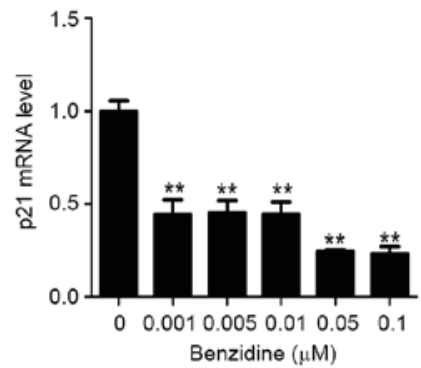

Figure 3. Benzidine exposure induced a change in the expression level of cell cycle markers. (A) Representative image of the western blotting analysis of cyclin D1, p21 and PCNA protein levels, with GAPDH as a loading control. The expression of cyclin D1 and PCNA was upregulated, whereas p21 was downregulated. (B) Densitometric analysis of the western blots. (C) Measurement of the mRNA level of cyclin D1, PCNA and p21 by reverse transcription-quantitative polymerase chain reaction; the same trend of cyclin D1 and PCNA upregulation, and p21 downregulation, was observed. The data are expressed as the mean \pm standard deviation of three independent experiments. ${ }^{*} \mathrm{P}<0.05,{ }^{* *} \mathrm{P}<0.01$ vs. control. PCNA, proliferating cell nuclear antigen.

A

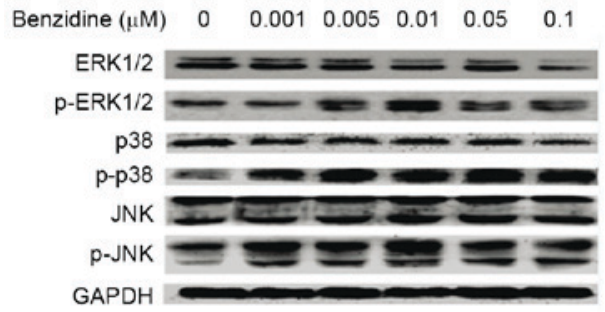

C

Benzidine ( $\mu$ M) $\quad 0 \quad 0.001 \quad 0.005 \quad 0.01 \quad 0.05 \quad 0.1$ p-c-jun $-\infty$

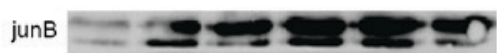

junD

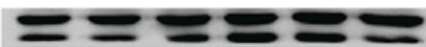

GAPDH

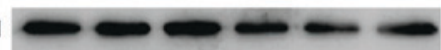

E

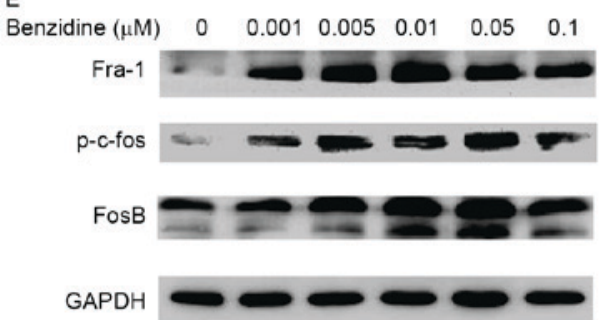

B

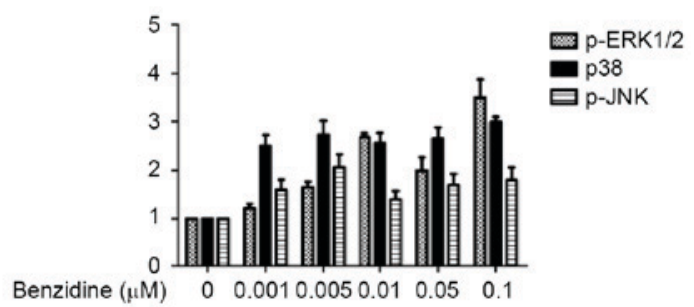

D

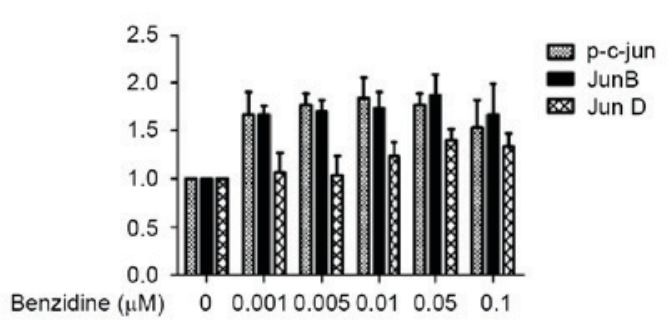

F

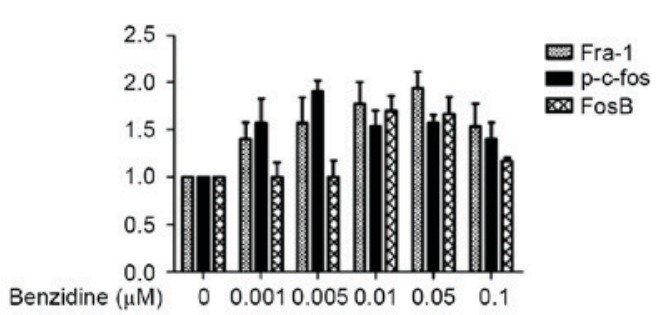

Figure 4. Effects of benzidine on the expression and activation of mitogen-activated protein kinases and activating protein-1 monomers. (A) Total and phosphorylated ERK1/2, p38 and JNK protein levels were determined by western blotting. The levels of p-ERK1/2, p-p38 and p-JNK increased without any significant changes to total ERK1/2, p38 or JNK levels, indicating that benzidine exposure activated ERK1/2, p38, and JNK. The alterations to p-ERK1/2, p-p38 and p-JNK protein level occurred particularly at concentrations of 0.005 or $0.01 \mu \mathrm{M}$ benzidine. (B) Densitometric quantification of the data from A. (C) Western blotting analysis of Jun family proteins. Significant increases in p-c-Jun and JunB levels were observed, whereas the JunD level was not significantly increased. (D) Densitometric quantification of the data from C. (E) The Fos family, including p-c-Fos, FosB and Fra-1, were all upregulated. (F) Densitometric quantification of the data from E. Densitometric data are expressed as the mean \pm standard deviations of three independent experiments. p-, phosphorylated; ERK1/2, extracellular regulated protein kinases 1 and 2; JNK, Jun N-terminal kinase; Fra-1, Fos-like antigen 1. 

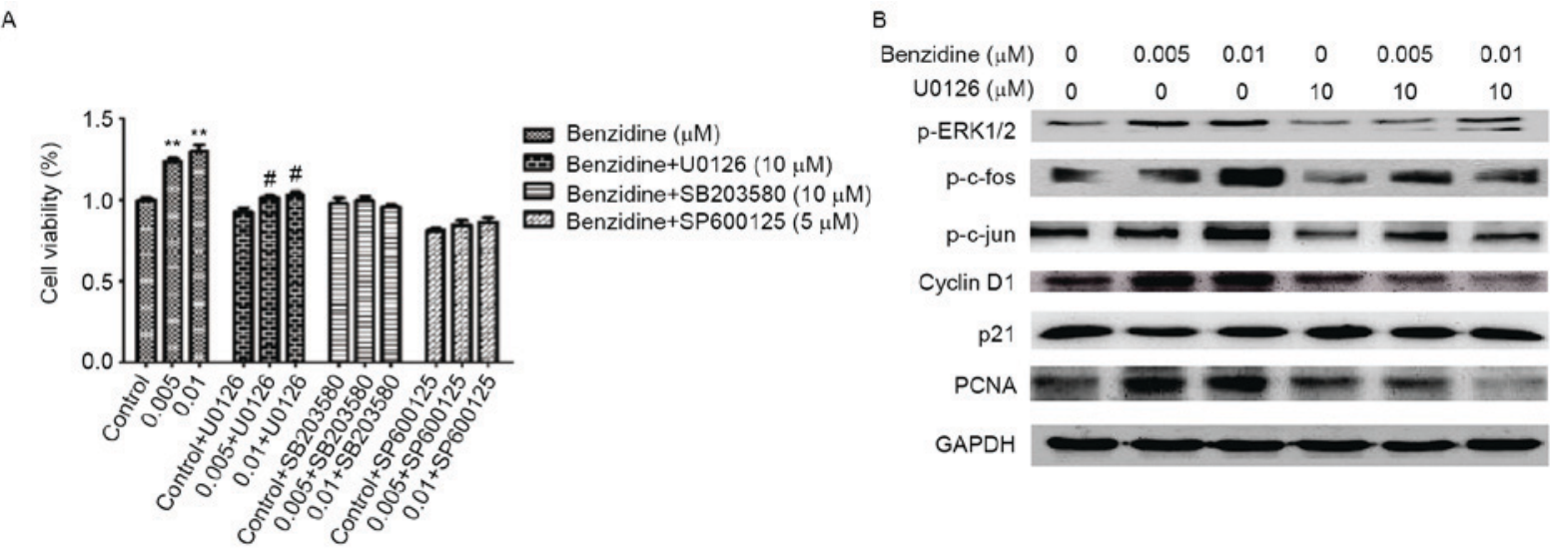

C
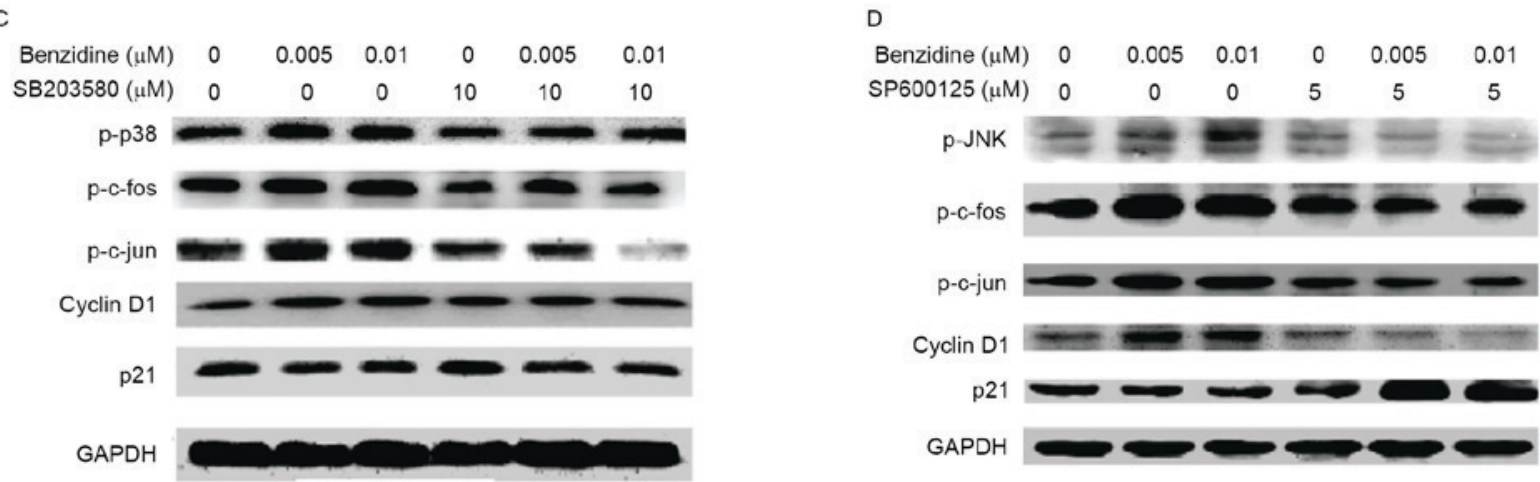

Figure 5. Proliferation-inducing effect of benzidine on SV-HUC-1 cells was reversed by MAPK inhibitors. (A) The relative cell proliferation was suppressed by MAPK pathway inhibitors. The effect of the $\mathrm{p} 38$ inhibitor was the most distinct, as no increase in cell proliferation following the treatment with benzidine was observed. Western blot of SV-HUC-1 cells treated with benzidine and (B) U0126, an ERK1/2 inhibitor, (C) SB203580, a p38 inhibitor or (D) SP600125, a Jun $\mathrm{N}$-terminal kinase inhibitor, for 6 days. Following the treatment with the MAPK inhibitors, the effect of benzidine treatment on cyclin D1 and p21 expression levels was suppressed; however, the increase in PCNA protein levels were inhibited only by U0126. Data is expressed as the means \pm standard deviation of three independent experiments for each treatment. ${ }^{* *} \mathrm{P}<0.01$ vs. control; ${ }^{*} \mathrm{P}<0.05$ vs. control+U0126. SV-HUC-1, SV-40 immortalized human uroepithelial cells; MAPK, mitogen-activated protein kinase; ERK1/2, extracellular regulated protein kinases 1 and 2; PCNA, proliferating cell nuclear antigen.

p21, a cyclin dependent kinase inhibitor in the G1/S transition, is a downstream mediator of tumor suppressor p53. It is a well-characterized partner of PCNA that has been identified as occurring in a complex of PCNA, cyclin D1 and cyclin-dependent kinases (CDKs). The p21 protein has two inhibitory effects on the entry of a cell into S-phase, including the inhibition of CDK kinase activity and the inhibition of DNA replication via interactions with PCNA (21). A previous report demonstrated that $\mathrm{p} 21$ expression was associated with a poor prognosis in patients with bladder cancer (22). Consistent with previous observations, the results of the present study revealed that benzidine-induced SV-HUC-1 cell proliferation was associated with the upregulation of cyclin D1 and PCNA, and the downregulation of $\mathrm{p} 21$.

Multiple signaling pathways are associated with the regulation of the cell cycle. MAPK pathways, which include a series of protein kinase cascades, serve important roles in various biological processes, including cell proliferation. The pathway associated with ERK1/2, MAPK family members, may induce the initiation and progression of cancer $(23,24)$. In the present study, it was identified that not only ERK1/2, but also p38 and JNK, were activated in benzidine-induced SV-HUC-1 cell proliferation. In addition, cell proliferation was reversed when MAPK-specific inhibitors were used together with benzidine, indicating the pivotal role of MAPK activation in benzidine-induced SV-HUC-1 cell proliferation.
AP-1 is a transcription-activating heterodimer composed of members of the Jun and Fos families. It is associated with cell proliferation and differentiation, and the invasion and metastasis of cancer (25). A previous study revealed that upregulation of AP-1 enhanced anaplastic large cell lymphoma progression and dissemination (26). In the present study, benzidine promoted the activation of AP-1 monomers and AP-1 was downregulated following the inhibition of MAPKs, with benzidine-induced cell proliferation reversed simultaneously. The results revealed that MAPKs regulated the benzidine-induced SV-HUC-1 cell proliferation via the regulation of AP-1.

In conclusion, the present study demonstrated that low concentrations of benzidine lead to increased cell proliferation via the upregulation of the MAPK/AP-1 pathway in SV-HUC-1 cells. The inhibition of MAPKs reversed benzidine-induced SV-HUC-1 proliferation. These findings indicate the role of MAPK pathways in benzidine-induced pathologies, including tumorigenesis, and may provide novel insights into the molecular mechanisms that underlie pathologies induced by benzidine or other aromatic amine compounds.

\section{Acknowledgements}

The present study was supported by grants from the National Natural Science Foundation of China (grant nos. 81373005, 
81072330 and 81202194 ) and by the Priority Academic Program Development of Jiangsu Higher Education Institutions.

\section{Funding}

The National Natural Science Foundation of China (Beijing, China; grant nos. 81373005, 81072330 and 81202194).

\section{Availability of data and materials}

The datasets used and/or analyzed during the current study are available from the corresponding author on reasonable request.

\section{Authors' contributions}

DXY and CYZ conceived and designed the study. LZ, TZ, HG, ZQL, ZFL, ZQZ and JM performed the experiments. LZ wrote the paper. LZ, TZ, HG, ZQL, ZFL, DXY and CYZ reviewed and edited the manuscript. All authors read and approved the manuscript.

\section{Ethics approval and consent to participate}

The Medical Ethics Committee of Anhui Medical University gave approval for this study, and all participants gave informed consent.

\section{Consent for publication}

Not applicable.

\section{Competing interests}

The authors declare they have no competing interests.

\section{References}

1. Cheung G, Sahai A, Billia M, Dasgupta P and Khan MS: Recent advances in the diagnosis and treatment of bladder cancer. BMC Med 11: 13, 2013.

2. Siegel RL, Fedewa SA, Miller KD, Goding-Sauer A, Pinheiro PS, Martinez-Tyson D and Jemal A: Cancer statistics for Hispanics/Latinos, 2015. CA Cancer J Clin 65: 457-480, 2015

3. Yu MC, Skipper PL, Tannenbaum SR, Chan KK and Ross RK: Arylamine exposures and bladder cancer risk. Mutat Res 506-507: 21-28, 2002.

4. Letašiová $S$, Dušinská $M$, Volkovová $K$, Mosoiu $C$ and Bartonva A: Bladder cancer, a review of the environmental risk factors. Environ Health 11 (Suppl 1): S11, 2012.

5. Zhao L, Geng H, Liang ZF, Zhang ZQ, Zhang T, Yu DX and Zhong CY: Benzidine induces epithelial-mesenchymal transition in human uroepithelial cells through ERK1/2 pathway. Biochem Biophys Res Commun 459: 643-649, 2015.

6. Correia I, Alonso-Monge R and Pla J: MAPK cell-cycle regulation in Saccharomyces cerevisiae and Candida albicans. Future Microbiol 5: 1125-1141, 2010.

7. Thomasova D and Anders HJ: Cell cycle control in the kidney. Nephrol Dial Transplant 30: 1622-1630, 2015.

8. Chang LF and Karin M: Mammalian MAP kinase signalling cascades. Nature 410: 37-40, 2001.
9. Sangrar W, Shi C, Mullins G, LeBrun D, Ingalls B and Greer PA: Amplified Ras-MAPK signal states correlate with accelerated EGFR internalization, cytostasis and delayed HER2 tumor onset in Fer-deficient model systems. Oncogene 34: 4109-4117, 2015.

10. Dhanasekaran DN and Johnson GL: MAPKs: Function, regulation, role in cancer and therapeutic targeting. Oncogene 26: 3097-3099, 2007.

11. Ansari KM and Das M: Skin tumor promotion by argemone oil/alkaloid in mice: Evidence for enhanced cell proliferation, ornithine decarboxylase, cyclooxygenase-2 and activation of MAPK/NF-kappaB pathway. Food Chem Toxicol 48: 132-138, 2010.

12. Xu T, Zhou M, Peng L, Kong S, Miao R, Shi Y, Sheng H and Li L: Upregulation of CD147 promotes cell invasion, epithelial-to-mesenchymal transition and activates MAPK/ERK signaling pathway in colorectal cancer. Int J Clin Exp Pathol 7: 7432-7441, 2014.

13. Livak KJ and Schmittgen TD: Analysis of relative gene expression data using real-time quantitative PCR and the 2(-Delta Delta C(T)) method. Methods 25: 402-408, 2001.

14. Baldi A, De Luca A, Esposito V, Campioni M, Spugnini EP and Citro G: Tumor suppressors and cell-cycle proteins in lung cancer. Pathol Res Int 2011: 605042, 2011.

15. Sobus SL and Warren GW: The biologic effects of cigarette smoke on cancer cells. Cancer 120: 3617-3626, 2014

16. Li X, Gong X, Chen J, Zhang J, Sun J and Guo M: miR-340 inhibits glioblastoma cell proliferation by suppressing CDK6, cyclin-D1 and cyclin-D2. Biochem Biophys Res Commun 460: 670-677, 2015.

17. Sun Y, Luo D and Liao DJ: CyclinD1 protein plays different roles in modulating chemoresponses in MCF7 and MDA-MB231 cells. J Carcinog 11: 12, 2012.

18. Guo ZY, Hao XH, Tan FF, Pei X, Shang LM, Jiang XL and Yang F: The elements of human cyclin D1 promoter and regulation involved. Clin Epigenetics 2: 63-76, 2011.

19. Malmström PU, Wester K, Vasko J and Busch C: Expression of proliferative cell nuclear antigen (PCNA) in urinary bladder carcinoma. Evaluation of antigen retrieval methods. APMIS 100: 988-992, 1992

20. Russo G, Zamparelli A, Howard CM, Minimo C, Bellan C, Carillo G, Califano L, Leoncini L, Giordano A and Claudio PP: Expression of cell cycle-regulated proteins pRB2/p130, p107, E2F4, p27, and pCNA in salivary gland tumors: Prognostic and diagnostic implications. Clin Cancer Res 11: 3265-3273, 2005.

21. Waga S, Hannon GJ, Beach D and Stillman B: The p21 inhibitor of cyclin-dependent kinases controls DNA replication by interaction with PCNA. Nature 369: 574-578, 1994.

22. Shariat SF, Kim J, Raptidis G, Ayala GE and Lerner SP: Association of $\mathrm{p} 53$ and p21 expression with clinical outcome in patients with carcinoma in situ of the urinary bladder. Urology 61: 1140-1145, 2003

23. Zhang YQ, Wei XL, Liang YK, Chen WL, Zhang F, Bai JW, Qiu SQ, Du CW, Huang WH and Zhang GJ: Over-expressed twist associates with markers of epithelial mesenchymal transition and predicts poor prognosis in breast cancers via ERK and Akt activation. PloS One 10: e0135851, 2015.

24. Li T, Zhang C, Ding Y, Zhai W, Liu K, Bu F, Tu T, Sun L, Zhu W, Zhou F, et al: Umbilical cord-derived mesenchymal stem cells promote proliferation and migration in MCF-7 and MDA-MB-231 breast cancer cells through activation of the ERK pathway. Oncol Rep 34: 1469-1477, 2015.

25. Han C and Wan F: Research progress on AP-1. Chin J Cell Biol, 2017.

26. Schiefer AI, Vesely P, Hassler MR, Egger G and Kenner L: The role of AP-1 and epigenetics in ALCL. Front Biosci (Schol Ed) 7: 226-235, 2015. 